\title{
Factors affecting the voluntary intake of food by sheep
}

\author{
4. The effect of additives representing the primary tastes on sham intakes by \\ oesophageal-fistulated sheep* \\ BY W. L. GROVUM AND H. W. CHAPMAN \\ Department of Biomedical Sciences, Ontario Veterinary College University of Guelph, \\ Guelph, Ontario NIG 2WI, Canada
}

(Received 3 March 1987-Accepted 28 May 1987)

\begin{abstract}
1. Sheep with oesophageal fistulas were sham-fed on pelleted lucerne (Medicago sativa) hay to assess the palatability of added chemicals representing the tastes of sweet (sucrose), sour (hydrochloric acid), salt (sodium chloride), bitter (urea) and umami (monosodium glutamate; MSG).

2. Plain pellets and four concentrations of each chemical were sham-fed for $30 \mathrm{~min}$ after $5.5 \mathrm{~h}$ deprivation in $5 \times 5$ Latin-square experiments following a period of adaptation feeding.

3. Sucrose, at concentrations of $15-120 \mathrm{~g} / \mathrm{kg}$ air-dried pellets, depressed intakes with a linear relation between intake $(\mathrm{I} ; \mathrm{g})$ and concentration $(\mathrm{C} ; \mathrm{g} / \mathrm{kg}): \mathrm{I}=1001-3 \cdot 42 \mathrm{C}$.

4. $\mathrm{HCl}$ at $6.25-25.0 \mathrm{~g} / \mathrm{kg}$ pellets had no effect on sham intakes but at $50 \mathrm{~g} / \mathrm{kg}$ reduced them by $50 \%$ of control $(P<0.05)$.

5. $\mathrm{NaCl}$ at $50-200 \mathrm{~g} / \mathrm{kg}$ increased sham intakes by $26 \%(P<0.01)$ with no evidence of a dose-related effect.

6. Urea at $10-80 \mathrm{~g} / \mathrm{kg}$ decreased sham intakes by $26.9 \%(P<0.01)$ with no evidence of a dose-related effect.

7. MSG at $5-40 \mathrm{~g} / \mathrm{kg}$ in two experiments increased sham intakes by 16.1 and $40.8 \%(P<0.05)$. In another experiment at $1-8 \mathrm{~g} / \mathrm{kg}$ there was no significant effect.

8. When palatability and post-ingestive effects are separated by sham-feeding, the effect of added chemicals on intake may be completely different from when they are ingested normally (e.g. $\mathrm{NaCl}$ and sucrose). This newly developed technique enables the palatability effect of feed additives to be tested critically and economically.
\end{abstract}

Palatability has been defined as the response of an organism to such qualities of a foodstuff as its taste, smell, texture and appearance (Young, 1967). The need to separate postingestive from cephalic stimuli has been stressed (Pfaffman, 1959; Greenhalgh \& Reed, 1967) but, despite the importance of palatability in ruminant nutrition (Grovum, 1987), there have been few studies of the effects of cephalic (or pre-rumen) stimuli uncomplicated by post-ingestive effects. That the senses of taste, smell and touch influence intake has been demonstrated by experiments in which nerves have been cut (Chapman, 1965; Arnold, 1966; Arnold et al. 1980). Single-fibre recording has shown that chemoreceptors in the sheep's tongue are sensitive to salt, sweet, sour and bitter (Iggo \& Leek, 1967).

Brief exposure tests have been suggested as an uncontaminated measure of palatability (Pfaffman, 1959), but these tests necessarily do not permit prolonged or repeated sampling and the cumulated small doses may have post-ingestive effects. Sham-feeding, in which the ingesta are diverted from the digestive tract through an oesophageal or gastric fistula, avoids these problems (Weingarten \& Watson, 1982). There have been no reports of the use of this technique in ruminants however, despite the well-established use of oesophageal fistulas to sample the forages eaten by grazing herbivores (Langlands, 1975).

The experiments reported in the present paper examine the effect on the palatability of sham-fed lucerne (Medicago sativa) pellets of five chemicals representing five primary tastes: sucrose (sweet), hydrochloric acid (sour), sodium chloride (salt), urea (bitter) and

* Part 1 appeared in the British Journal of Nutrition (1978) 40,323-336; part 2 in the British Journal of Nutrition (1979) 42, 425-436; part 3 in the British Journal of Nutrition (1981) 45, 183-201. 
umami (monosodium glutamate (MSG); Cagan, 1977). Although this classification of tastes is based on human subjective responses there is some evidence that it may apply to animals as well (Jacobs et al. 1978). Brief reports on parts of the work described here have been published previously (Grovum \& Chapman, 1982; Chapman \& Grovum, 1984).

\section{EXPERIMENTAL}

\section{Sheep, housing and feeding}

Nineteen crossbred Suffolk sheep had oesophageal fistulas established. Twelve of these were 5 -month-old wether lambs, two were 12 -month-old wethers and five were culled ewes ages $2-3$ years at the time of surgery. The six sheep in current experimental use were housed in metabolism cages in one room and spares were kept in pens in another room. Both rooms were air-conditioned and continuously lit and a radio was left on to provide background noise. Water was always available. Lucerne hay was given ad lib. and mineral blocks were provided except during the deprivation and experimental periods.

The results of analysis of the lucerne hay were $(\mathrm{g} / \mathrm{kg}$ dry matter): crude protein 202 , acid-digestible fibre 314 , fat 22 , ash 66 . The sheep were treated for internal parasites with subcutaneous injections of a broad-spectrum anthelmintic (Tramisol; Cyanamid) and for external parasites by dusting with a powder containing malathion. After deaths attributed to chronic copper poisoning occurred, treatments with sodium molybdate were carried out. Foot trimming and shearing were done when needed and sheep were periodically removed from the cages and kept in the pens for exercise.

\section{Oesophageal fistulas, plugs and sample collection}

Before surgery each sheep was deprived of food for $24 \mathrm{~h}$. Halothane anaesthesia at a plane deep enough to eliminate swallowing was used and the sheep was placed in dorsal recumbency with the neck stretched out. The ventral surface of the neck was prepared for aseptic surgery and a smooth-tipped stomach tube was passed into the oesophagus. A longitudinal $70-100 \mathrm{~mm}$ skin incision was made over the tip of the tube (usually $30-50 \mathrm{~mm}$ left of the midline), the underlying muscles were divided by blunt dissection and the oesophagus was exposed using the stomach tube as a guide. An incision was made with a scalpel through the oesophageal wall on to the tube, thus avoiding the possibility of dissecting laterally into the wall of the oesophagus. The full thickness of the wall of the oesophagus was grasped with two pairs of foreceps and the incision enlarged with scissors longitudinally so that the plug to be used would fit snugly. The oesophageal wall was sutured to the skin ( 000 gauge silk attached to an atraumatic needle) and the remainder of the skin incision was closed. A split-rubber plug was inserted and a dose of penicillin and streptomycin injected intramuscularly.

The plug design (Plate 1) was modified from that of Chapman (1964). The plugs (Chapman \& Grovum, 1984) were made in various sizes from heat-sensitive polyvinyl chloride (Plastisol Base CA 106; F. H. and Sons, Toronto, Canada) which was poured into an aluminium mould and heated in an oven. After removal from the mould the plugs were cut in half down the middle of the stem and some of the stem was hollowed out to reduce its weight. After insertion of the flanges into the oesophagus through the fistula, the two halves of the stem were held together by an external rubber washer (about $100 \mathrm{~mm}$ in diameter, $10 \mathrm{~mm}$ thick and with a central hole tailored to fit the plug) and a rubber taildocking ring.

During sham-feeding the plugs were removed and sampling bags attached to the necks of the animals. These bags were made from rubber rectal examination sleeves with attached rubber straps which were fastened around the neck of the animals. The straps were adjusted 
so that the shoulder end of the sleeve was below the opening of the fistula while the sheep had its head down for feeding. The hand end of the sleeve was cut off and was closed with a tail-docking ring. At the end of the feeding period the bags were removed and emptied.

\section{Post-operative maintenance}

The sheep were inspected twice daily and each day the neck was washed using a pressure hose and a mild detergent. The wool on the neck was clipped each week. If the fistula enlarged or contracted spontaneously the plug size was changed. The fistulas shrank if the plug was out for a long time but could usually be enlarged by inserting successively larger plugs. If this was unsuccessful an incision was made (using local anaesthesia) at the cranial or caudal end of the fistula and a larger plug inserted.

The criterion for the use of a sheep in an experiment was that $80-100 \%$ of a pellet meal could be recovered from the fistula. This was usually met by fistulas fitted with plugs $100 \mathrm{~mm}$ or more in diameter. To measure the recovery of the pellets fed, the material extracted from the fistula was weighed and samples were dried in an oven to constant weight.

Fistulas that became too large leaked excessively and were partially closed by excising the margin of the cranial or caudal end of the fistula under local or halothane anaesthesia. The edges of the oesophagus exposed by this cut were sutured together and the cut edges of the skin were closed taking care to ensure a good seal at the junction of the remaining fistula and the closed section.

The most serious complication after surgery was oesophageal obstruction. This occurred frequently if the sheep were fed on pellets with the plugs in place, and was the cause of death of one sheep. For this reason, hay was used for maintenance feeding of the sheep. The attendants watched for signs of obstruction, including inappetance, palpable masses in the neck and frothy saliva around the mouth and the fistula. Obstructions were easily cleared with the fingers or by passing a stomach tube through the fistula. Occasionally sheep developed acidosis, presumably as a result of the chronic loss of saliva through the fistula. The signs were inappetance and depression and the diagnosis was confirmed by blood-gas analysis. The treatment was $20 \mathrm{~g}$ sodium bicarbonate in 1 litre water administered intraruminally. $\mathrm{NaCl}$ was available ad lib. (in the mineral blocks) to avoid sodium depletion from the slight leakage of saliva through the fistulas.

Six of the twelve lambs operated on were kept in good condition for at least 18 months after surgery. One of the initial deaths was due to pharyngeal obstruction by feed pellets and two were related to repeated enlargement of the fistulas. The other three early deaths and those occurring after 18 months were not related to the fistulation procedure. During the first 3 months after surgery on the lambs, considerable difficulty was experienced and surgery to close enlarged fistulas was frequently necessary. This problem, presumably caused by the more elastic connective tissue of young animals, might have been avoided if smaller fistulas had been made initially. Fewer difficulties were encountered when older sheep were fistulated. One of the seven older sheep had recurring obstruction of the oesophagus, associated with a very large fistula and requiring complete closure of the fistula, another had lung abscesses and was euthanized and the remaining five were used for over 2 years.

The adequacy of the maintenance procedures and the diet and the benign nature of the established fistulas and the experimental procedure are indicated by the fact that the animals remained in good health and gained weight over the experimental period. Except during occasional blockages of the oesophagus the sheep were able to swallow hay and to regurgitate normally. The infrequent cases of acidosis and the blockages were easily treated 
and the spontaneous deaths occurring after the initial post-operative period were the result of $\mathrm{Cu}$ poisoning. The maintenance problems that complicated the research were the variations in the size of the fistulas and the fluctuating intakes which are common in grown sheep on full feed.

\section{Experimental protocol}

On experimental days the sheep to be used were fed on fresh hay from 08.00 to 09.00 hours then deprived of food for $5.5 \mathrm{~h}$. At 14.15 hours the oesophageal plugs were removed and the sampling bags were attached. The sheep were offered weighed amounts of pellets for three 10 min periods from 14.30 hours and the intakes recorded for each period. The total intake for the three periods was used in the analysis of results. At the end of the experiment the bags were removed, the plugs replaced and the hay and the salt blocks returned to the sheep. Before being used in any experiment the sheep were sham-fed on plain lucerne pellets for at least $5 \mathrm{~d}$ to accustom them to the experimental routine. A period of adaptation shamfeeding using plain pellets was also provided whenever a new technician was employed or when a new sheep was brought into the experimental room.

\section{Experiments}

The various concentrations of the additives tested (Table 1) were mixed with lucerne meal using a mechanical mixer and pelleted in $4.7 \mathrm{~mm}$ pellets. The additives were laboratory grade chemicals, except sucrose which was commercial grade. The pellets containing $\mathrm{HCl}$ were stored in sealed plastic bags until used. Each additive to be used was sham-fed at one of the concentrations to be tested for an adaptation period lasting until the intakes of at least five sheep had reached an apparent plateau as determined by inspection of the results. These sheep were then used in a $5 \times 5$ Latin square dose-response experiment. The ranges used for the concentrations of the test chemicals in the Latin-square experiments were in some cases changed from those originally planned after analysis of the adaptation results. As a result the adaptation doses do not fall at the same points on the test-dose scale. The dose-response experiment using MSG was repeated with a higher series of concentrations immediately after the first experiment. Some time later the experiment was again repeated at the higher concentrations but on this occasion data were available from only four sheep.

\section{Statistical analysis}

The analysis of the adaptation results was by means of a $t$ test for the significance of the difference between the mean intakes for the first and last $3 \mathrm{~h}$ of the adaptation periods. The dose response data were subjected to analysis of variance for a $5 \times 5$ Latin square. This design was adopted to minimize the number of surgically prepared animals used. Possible problems with the design include heterogeneity of variance and carryover effects. There was no evidence in the adaptation periods of marked differences in the variability of the responses of the sheep. Carryover effects were not considered likely to be important since the sheep were not ingesting the chemicals and since they were adapted to the palatability effects. For those experiments in which the $F$ test was significant the differences between the means was tested using the sequential (NK) variant of the Q method (Snedecor \& Cochran, 1980 , p. 235). In addition, in each experiment the mean of the intakes of the plain pellets was compared with the mean of the intakes for all four levels of additives (a planned comparison, Snedecor \& Cochran, 1980, p. 224). Since inspection of the data suggested a linear relation between sucrose concentration and feed intake a regression analysis was performed on the sucrose data (Snedecor \& Cochran, 1980, p. 270). 
Table 1. Concentrations of additives used in adaptation periods and dose-response experiments $(\mathrm{g} / \mathrm{kg}$ air-dried pellets)

\begin{tabular}{|c|c|c|c|c|c|c|c|}
\hline \multirow[b]{2}{*}{ Taste } & \multirow[b]{2}{*}{ Chemical } & \multirow[b]{2}{*}{ Adaptation periods } & \multicolumn{5}{|c|}{ Dose-response treatments } \\
\hline & & & 0 & 1 & 2 & 3 & 4 \\
\hline Sweet & Sucrose & 60 & 0 & 15 & 30 & 60 & 120 \\
\hline Sour & Hydrochloric acid & $12 \cdot 5$ & 0 & $6 \cdot 25$ & $12 \cdot 5$ & 25 & 50 \\
\hline Salt & Sodium chloride & 100 & 0 & 50 & 100 & 150 & 200 \\
\hline Bitter & Urea & 10 & 0 & 10 & 20 & 40 & 80 \\
\hline Umami & MSG: a & $2 \cdot 0$ & 0 & 1.0 & $2 \cdot 0$ & $4 \cdot 0$ & 8.0 \\
\hline & $\mathrm{b}$ & - & 0 & $5 \cdot 0$ & 10 & 20 & 40 \\
\hline & $c$ & $5 \cdot 0$ & 0 & $5 \cdot 0$ & 10 & 20 & 40 \\
\hline
\end{tabular}

MSG, monosodium glutamate.

\section{RESULTS}

\section{Adaptation feeding}

The first six sheep used were sham-fed on lucerne pellets on each of $10 \mathrm{~d}$ to examine the effect of adaptation to the experimental regimen and the pellets. The mean intakes ( $\mathrm{g}$ during the 30 min sham-feeding period) were: $488,605,644,589,743,985,826,791,712$ and 849 , with the difference between the aggregated means for the first and the last $3 \mathrm{~d}$ (579 and $784 \mathrm{~g}$ respectively) being significant $(P<0.05$, paired $t$ test). Sheep added subsequently were also sham-fed on plain lucerne pellets to adapt them to the experimental regimen but the values are not comparable since the sheep varied in size and age and were fed next to sheep already adapted.

The results for the adaptation periods for each of the additives tested are shown in Table 2 which lists the mean intakes for the sheep subsequently used in the Latin-square tests. The intakes of pellets containing sucrose fell, during adaptation sham-feeding, from a mean of $1586 \mathrm{~g}$ for the first $3 \mathrm{~d}$ to a mean of $337 \mathrm{~g}$ in the last $3 \mathrm{~d}(P<0.005$, paired $t$ test), while when $\mathrm{NaCl}$ was added the intake of pellets increased from 733 to $1410 \mathrm{~g}(P<0.01)$. No changes occurred in mean intakes during adaptation to $\mathrm{HCl}$, urea or MSG. No adaptation period was necessary for the second MSG experiment since it was conducted immediately after the first, using the same sheep. For the third MSG experiment the mean intakes for three successive days of adaptation feeding were 1312,1873 and $1391 \mathrm{~g}(n 4)$. Control intakes of plain pellets for comparison with the intakes of the treated pellets were not obtained since the periods of adaptation feeding were intended only to eliminate any possible response of the sheep to novel tastes.

\section{Effect of additives}

All the additives had a significant effect on the intakes of sheep sham-feeding on lucerne pellets (Table 3). Analysis of variance revealed a significant treatment effect for sucrose $(P<0.05), \mathrm{HCl}(P<0.005)$ and urea $(P<0.005)$, all of which reduced intakes. At the highest concentration of sucrose $(120 \mathrm{~g} / \mathrm{kg})$ sham intakes were significantly less than that for plain pellets $(P<0.05)$ and the mean for all the concentrations of sucrose, combined, was also significantly less than that of plain pellets. There was also a significant $(P<0.05)$ linear relation between sucrose concentration ( $\mathrm{C} ; \mathrm{g}$ sucrose $/ \mathrm{kg}$ pellets) and intake (I;g pellets) with $\mathrm{I}=1001-3 \cdot 42 \mathrm{C}$. In contrast urea depressed intakes approximately equally at all the 


(1)


concentrations used $(10-80 \mathrm{~g} / \mathrm{kg})$. A third pattern appeared with $\mathrm{HCl}$ addition. Only the highest concentration of $\mathrm{HCl}(50 \mathrm{~g} / \mathrm{kg})$ depressed intakes significantly.

MSG (at the higher range of concentrations) and $\mathrm{NaCl}$ increased intakes when added to sham-fed pellets. These effects were significant $(P<0.05)$ when the mean intake for all concentrations of each additive was compared with that for plain pellets. For MSG the effect appeared to be dose-dependent increasing to a maximum at $10 \mathrm{~g} / \mathrm{kg}$ and declining slightly at 20 and $40 \mathrm{~g} / \mathrm{kg}$, but these differences were not significant. There was no evidence of a concentration effect with $\mathrm{NaCl}$ addition.

\section{DISCUSSION}

The intake of food is determined by the complex interaction of pre- and post-absorptive factors (Baile \& Forbes, 1974). Those factors operating before the food leaves the mouth can be collectively referred to as palatability (Young, 1967) while those operating after this stage are referred to here as post-ingestive. The present paper shows that palatability and post-ingestive effects can be separated and is the first report of such separation in ruminants. The need for this separation and the value of this approach are illustrated by the $\mathrm{NaCl}$ findings. When $\mathrm{NaCl}$ is included in the diet it depresses intake (Wilson, 1966). That this is the result of post-ingestive effects is shown by the increase in intake when $\mathrm{NaCl}$ is sham-fed (Table 3).

The separation of palatability from post-ingestive effects is complicated by the effects of novelty and of learning on intake. While information on sheep is lacking, research in other species has shown the importance of novelty per se, or of previously acquired associations between taste and post-ingestive factors (Rozin, 1976). During sham-feeding, intakes may be initially enhanced or depressed by these effects. The adaptation periods were intended to eliminate any confounding effects of experience or novelty, rather than to identify them, and therefore did not include controls for temporal or environmental effects. Thus the intake changes during the adaptation periods for sucrose and $\mathrm{NaCl}$ may reflect environmental or other variables or may indicate that learned associations influenced the intakes of pellets containing these additives. More research is clearly required in this area.

The dose-response values show that $\mathrm{NaCl}$ pellets were preferred to plain pellets at all concentrations when sham-fed (Table 3). Since these sheep had ad lib. access to salt they were unlikely to have been $\mathrm{Na}$ deficient. In Na-replete sheep $\mathrm{NaCl}$ in feed reduced intake (Wilson, 1966) and water was preferred to a $\mathrm{NaCl}$ solution when the concentration of the $\mathrm{NaCl}$ solution exceeded $12.5 \mathrm{~g} / 1$ (Goatcher \& Church, 1970b). The increased intake found with sham-feeding (Table 3) indicates that this rejection is the result of the post-ingestive effects of the $\mathrm{NaCl}$. The adaptation findings (Table 2) may indicate that, in sheep feeding normally, an association is established between the taste of $\mathrm{NaCl}$ and these post-ingestive effects and that this association is extinguished by sham-feeding. The experimental sheep had had ample opportunity to form such an association since $\mathrm{NaCl}$, unlike the other additives tested, was included in the maintenance diet.

Sucrose reduced the intake of sham-fed lucerne pellets at each concentration, the reduction being statistically significant for the highest concentration and for the mean value for all concentrations (Table 3). These findings show that, in the absence of post-ingestive effects, sucrose is relatively unpalatable to sheep. In contrast, when sucrose was ingested normally, there was no evidence of discrimination between sucrose solutions $(8-200 \mathrm{~g} / \mathrm{l})$ and water except at the highest concentration (Goatcher \& Church 1970 b). Furthermore, when a less adequate diet was given, sucrose was preferred to water (Church \& Randall, 1979). This interaction of nutritional state and sucrose preference supports the importance of post-ingestive effects. The possible existence of a learned preference for sweet taste, based 
on these effects, is indicated by the fall of intake during the period of adaptation to pellets with sucrose (Table 2).

The reduced intake by ruminants of feedstuffs containing added urea may be the result of either its bitter taste or its post-ingestive effects, including an increase in rumen and plasma ammonia levels. Attempts to differentiate between these explanations, by comparing the effects of oral and intraruminal urea in cattle, have been inconclusive (Huber \& Cook, 1972; Martz et al. 1973). The results presented in Table 3 show that urea depressed intake when sham-fed. Thus the effect of urea on feed intake, of sheep feeding normally, is at least in part due to its bitter taste. Post-ingestive effects may make an additional contribution. During the adaptation period intakes of the urea pellets did not change but since there is no evidence that the experimental sheep had previously been fed on urea no speculation on the existence of a conditioned response to the bitter taste of the urea is possible.

The sham intake of pellets containing $\mathrm{HCl}$ was significantly depressed only at the highest concentration (Table 3). The extremely low pH of the ingesta after it was wetted by saliva may explain the depression at this concentration. When $\mathrm{HCl}$ was added to water, intake was depressed at much lower concentrations (Goatcher \& Church, 1970a). The difference between the results can be attributed in part to the post-ingestive effects and in part to the fact that acid mixed with feed is buffered by the feed and must be dissolved by saliva (which also contains buffers) before it can stimulate the taste buds and other receptors. In contrast when acid is dissolved in water it has direct access to the receptors, less saliva is produced and the saliva is diluted by the water.

The effect of MSG depended on the concentration. At the lower range of concentrations $(1-8 \mathrm{~g} / \mathrm{kg})$ MSG had no effect on intake but at the higher range $(5-40 \mathrm{~g} / \mathrm{kg}) \mathrm{MSG}$ significantly increased the intake of sham-fed pellets. Since the concentration used in the adaptation period fell in the lower range the failure of intake to change during adaptation is inconclusive. The failure to obtain a response at the lower range of concentrations was unexpected since the only published findings on ruminants indicated that calves showed an initial preference to $2 \mathrm{~g}$ MSG $/ \mathrm{kg}$ when given a choice between creep feed with and without MSG (Waldern \& Van Dyk, 1971).

Previous studies of palatability in ruminants have failed to consider adequately the interaction of post-ingestive effects with palatability. Many have ignored this interaction and have claimed to measure the palatability of materials by simply adding them to feed or, more commonly, water (e.g. Arnold et al. 1980; Church \& Randall, 1979). At best, attempts have been made to estimate the post-ingestive effects by comparing the effect of feeding materials with those of administering them intraruminally (Greenhalgh \& Reid, 1967; Huber \& Cook, 1972).

Unfortunately the confounding of post-ingestive effects with palatability is not eliminated by this method which therefore does not give a pure measure of palatability. In other studies the role of the cranial senses has been assessed by measuring intake after the surgical removal of one or more of these senses (Chapman, 1965; Arnold et al. 1980) or when local anaesthetic has been added to the feed (Baile \& Martin, 1972). These approaches cannot measure the importance of the cranial senses as a whole and provide only a minimum estimate of the importance of the sense(s) removed since the animal may compensate for sensory deprivation by using senses not normally involved in intake, or by acquiring new associations between input from the unaffected senses and post-ingestive feedback.

In addition to avoiding post-ingestive effects the sham-feeding technique has the advantage of enabling additives to be incorporated with feeds, as well as with water. Differences in the vehicle used may partly account for differences between the current results and those reported, for example, by Goatcher \& Church $(1970 \mathrm{a})$. In the study of the effects of feed additives on palatability it is clearly desirable that the additives be incorporated with feed rather than with water since the controls of the intake of water may operate very 
differently from those of feed. Although grains were not fed to the oesophageal-fistulated sheep it seems probable that they would pass out of the fistula easily and would therefore also be suitable for sham-feeding.

The work is clearly relevant to the livestock-feeding industry. The palatability effects of many additives can now be tested critically, at least in sheep. A particular advantage of this technique for testing palatability effects is that the adaptation and testing periods can be short (since adaptation to the post-ingestive effects is not needed), enabling economical testing of large numbers of substances. Where palatability per se has an effect on intake appropriate action may be taken, for instance the search for an effective taste-masking agent for urea. Further work using this technique is now required, particularly with cattle, which are known to respond differently from sheep in intake tests using chemicals dissolved in water (Goatcher \& Church, 1970c).

The excellent technical assistance of Janette Wood and Barbara Gordon made this research possible. Funding was provided by the National Sciences and Engineering Research Council of Canada (Grant A2377 to W. L. G.) and by the Ontario Ministry of Agriculture and Food.

\section{REFERENCES}

Arnold, G. W. (1966). Australian Journal of Agricultural Research 17, 531-542.

Arnold, G. W., deBoer, E. S. \& Boundy, C. A. P. (1980). Australian Journal of Agricultural Research 31, $571-587$.

Baile, C. A. \& Forbes, J. M. (1974). Physiological Reviews 54, 160-213.

Baile, C. A. \& Martin, H. F. (1972). Journal of Dairy Science 55, 1461-1463.

Berridge, K., Grill, H. J. \& Norgren, R. (1981). Journal of Comparative and Physiological Psychology 95, $363-378$.

Cagan, R. H. (1977). In The Chemical Senses and Nutrition, pp. 343-360 [M. R. Kare and O. Maller, editors]. New York: Academic Press.

Chapman, H. W. (1964). Australian Veterinary Journal 40, 64-66.

Chapman, H. W. (1965). The anatomy and surgical deprivation of the oral and nasal senses in the sheep. MSc Thesis, Kansas State University.

Chapman, H. W. \& Grovum, W. L. (1984). Canadian Journal of Animal Science 64, Suppl., $106-107$.

Church, D. C. \& Randall, R. P. (1979). Journal of Animal Science 48, 1177-1181.

Goatcher, W. D. \& Church, D. C. (1970a). Journal of Animal Science 30, 784-790.

Goatcher, W. D. \& Church, D. C. (1970 b). Journal of Animal Science 31, 364-372.

Goatcher, W. D. \& Church, D. C. $(1970 \mathrm{c})$. Journal of Animal Science 31, 973-981.

Greenhalgh, J. F. D. \& Reid, C. W. (1967). Nature 214, 744.

Grovum, W. L. (1987). In The Ruminant Animal, pp. 202-216 [D. C. Church, editor]. Englewood Cliffs, New Jersey: Prentice Hall.

Grovum, W. L. \& Chapman, H. W. (1982). Proceedings of the Nutrition Society 41, 72A.

Hanson, H. L., Brushway, M. J. \& Lineweaver, H. (1960). Food Technology 14, 320 327.

Huber, J. T. \& Cook, R. M. (1972). Journal of Dairy Science 55, 1470-1473.

Iggo, A. \& Leek, B. F. (1967). In Olfaction and Taste, vol. 2, pp. 493-507. [T. Hayashi, editor]. London: Pergamon Press.

Jacobs, W. W., Beauchamp, G. K. \& Kare, M. R. (1978). In Flavor Chemistry of Animal Foods, pp. 1-20 [R. W. Bullard, editor]. Washington DC: American Chemical Society.

Langlands, J. P. (1975). In Digestion and Metabolism in the Ruminant, pp. 320-332 [I. W. McDonald and A. C. I. Warner, editors]. Armidale: University of New England Publishing Unit.

Martz, F. A., Wilson, G., Campbell, J. R. \& Hilderbrand, E. S. (1973). Journal of Animal Science 37, 351.

Pfaffman, C. (1959). In Handbook of Physiology Section 1, vol. 1, pp. 507-533 [H. W. Magoun, editor]. Washington, DC: American Physiological Society.

Rozin, P. (1976). In Advances in the Study of Behavior, vol. 6, pp. 21-76 [J. S. Rosenblatt, R. A. Hinde, E. Shaw and C. Beer, editors]. New York: Academic Press.

Snedecor, G. W., Cochran W. G. (1980). Statistical Methods, 7th ed. Ames: Iowa State University Press.

Waldern, D. E. \& Van Dyk, R. D. (1971). Journal of Dairy Science 54, 262-265.

Weingarten, H. P. \& Watson, S. D. (1982). Physiology and Behavior 28, 401-407.

Wilson, A. D. (1966). Australian Journal of Agricultural Research 17, 503-514.

Young, P. T. (1967). In Handbook of Physiology, Section 6, vol. 1, pp. 353-366 [C. F. Code, editor]. Washington, DC: American Physiological Society. 


\section{EXPLANATION OF PLATE}

Plate 1. A plug for an oesophageal fistula, made from heat-sensitive polyvinyl choloride using an aluminium mould. After removal from the mould the plug was cut in half and part of the stem hollowed out to reduce the weight of the plug. (Reprinted, by permission, from Chapman \& Grovum (1984).) 
British Journal of Nutrition, Vol. 59 No. 1

Plate 1

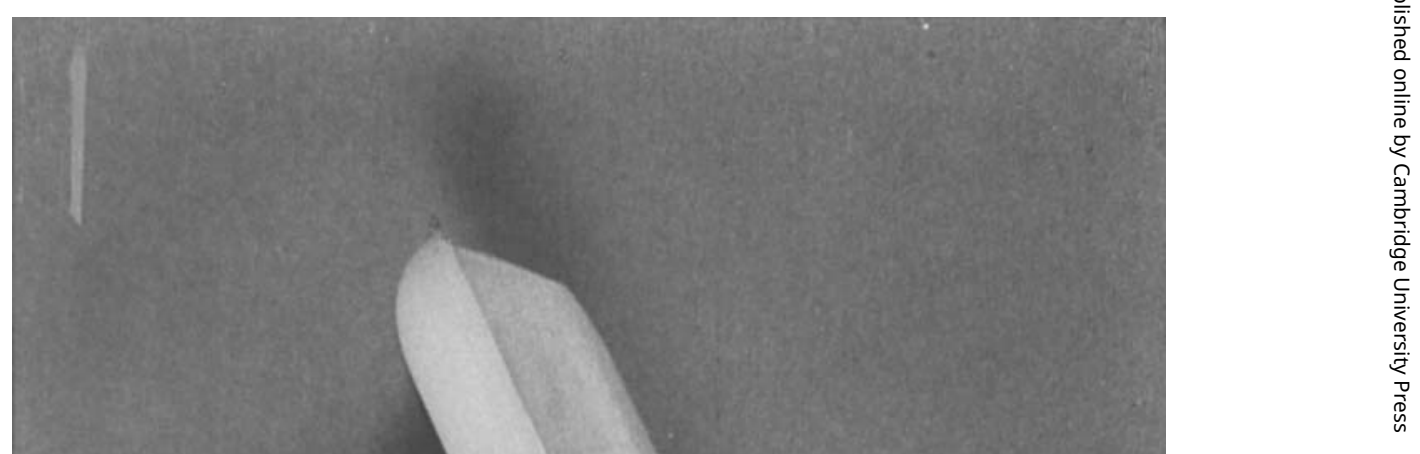

